\title{
A Sufficient Completeness Reasoning Tool for Partial Specifications Technical Report (Draft)
}

\author{
Joe Hendrix $^{1}$, Manuel Clavel$^{2}$, and José Meseguer ${ }^{1}$ \\ ${ }^{1}$ University of Illinois at Urbana-Champaign, USA \\ ${ }^{2}$ Universidad Complutense de Madrid, Spain
}

\begin{abstract}
We present the Maude sufficient completeness tool, which explicitly supports sufficient completeness reasoning for partial conditional specifications having sorts and subsorts and with domains of functions defined by conditional memberships. Our tool consists of two main components: (i) a sufficient completeness analyzer that generates a set of proof obligations which, if discharged, ensures sufficient completeness; and (ii) Maude's inductive theorem prover (ITP) that is used as a backend to try to automatically discharge those proof obligations.
\end{abstract}

\section{Introduction}

In computer science practice, equational specifications are often partial. That is, some of the relevant operations are only defined on an adequate subset of data. Simple examples of undefinedness include computing the top of an empty stack, division by zero, and many operations on data structures [1]. This has led to the design of increasingly more expressive equational formalisms to deal with partiality (see [2] for a survey). In particular, the papers [2-4] proposed membership equational logic (MEL) as a framework logic for the equational specification of partial functions. The key idea is that the domain of definition of a partial function is axiomatized by conditional membership axioms stating when the function is defined. However, since conditional memberships may have arbitrarily complex conditions and equations may be conditional, in this setting the sufficient completeness problem is undecidable in general.

The Maude sufficient completeness tool (SCC), which analyzes MEL theories specified in Maude, is therefore not a decision procedure. Instead it is a reasoning tool consisting of two main components: (i) a sufficient completeness analyzer that generates a set of proof obligations which, if discharged, ensures sufficient completeness of confluent, sort decreasing and reductive specifications; (ii) Maude's inductive theorem prover (ITP), that is used as a backend to try to automatically discharge those proof obligations.

Our tool has a number of useful applications. Two obvious ones are: (i) checking that the defined functions of a MEL specification will always evaluate to data built with the constructors; and (ii) for inductive theorem proving purposes, ensuring the correctness of the chosen proof technique (e.g. structural induction, 
cover set induction, inductionless induction, etc.) which typically depends on sufficient completeness. There are two other applications for which our tool has proved useful: (iii) checking that a rewrite theory specifying a concurrent system is deadlock-free, which is needed for verifying temporal logic properties using abstraction techniques [5]; the point is that deadlock-freeness can be characterized as the sufficient completeness of an associated MEL specification; and (iv) supporting more powerful cover set induction schemes in the style of [6] that can prove general conjectures of the form $\varphi\left(f\left(t_{1}, \ldots, t_{n}\right)\right)$, where $\varphi$ is a formula containing the expression $f\left(t_{1}, \ldots, t_{n}\right)$ with $f$ a defined function symbol and the $t_{1}, \ldots, t_{n}$ constructor terms; the point here is that the sufficient completeness checker can be used to generate base cases in the induction scheme which are needed because in general the $t_{1}, \ldots, t_{n}$ may be nonvariable terms [7]. This last application is a "turning of the tables" in the interoperation between Maude's ITP and SCC: in the second tool, the ITP plays an auxiliary role in discharging proof obligations, whereas in the ITP itself (which supports cover set induction) the sufficient completeness tool plays an auxiliary role in generating induction schemes.

\section{Preliminaries}

A MEL signature $\Sigma$ is a triple $\Sigma=(\mathcal{K}, \Sigma, \mathcal{S})$, where $\mathcal{K}$ is a set of kinds, $\mathcal{S}$ is a disjoint $\mathcal{K}$-kinded family $\mathcal{S}=\left\{\mathcal{S}_{k}\right\}_{k \in \mathcal{K}}$ of sets of sorts, and $\Sigma=\left\{\Sigma_{w, s}\right\}_{(w, s) \in \mathcal{K}^{*} \times \mathcal{K}}$ is a $\mathcal{K}$-kinded signature of function symbols. Given a $\mathcal{K}$-kinded disjoint family of finite sets of variables $\vec{x}=x_{1}: k_{1}, \ldots, x_{n}: k_{n}$, where $x_{1}, \ldots x_{n}$ are disjoint from the constants in $\Sigma$ and the kinds $k_{1}, \ldots k_{n}$ in the list can be repeated, a $\Sigma$-equation is a formula $t=t^{\prime}$, with $t, t^{\prime} \in T_{\Sigma}(\vec{x}), T_{\Sigma}(\vec{x})$ being the free $\Sigma$-algebra on the variables $\vec{x}$, and such that $t, t^{\prime}$ have the same kind, i.e. $t, t^{\prime} \in T_{\Sigma}(\vec{x})_{k}$ for some $k \in \mathcal{K}$. A $\Sigma$-membership is a formula $t: s$ such that if $t \in T_{\Sigma}(\vec{x})_{k}$, then $s \in \mathcal{S}_{k} . \Sigma$-sentences are universally quantified Horn clauses of the form

$$
(\forall \vec{x}) A \text { if } A_{1} \wedge \cdots \wedge A_{n}
$$

where $A$ and the $A_{i}$ are either $\Sigma$-equations or $\Sigma$-memberships. If $A$ is a $\Sigma$ equation, we call the sentence a conditional equation; and if $A$ is a $\Sigma$-membership, we call it a conditional membership. A MEL theory is a pair $\mathcal{E}=(\Sigma, \Gamma)$ with $\Sigma$ a MEL signature and $\Gamma$ a set of $\Sigma$-sentences. A model of a MEL signature $(\mathcal{K}, \Sigma, \mathcal{S})$ is a $(\mathcal{K}, \Sigma)$-algebra $\mathcal{A}$ together with a subset $\mathcal{A}_{s} \subseteq \mathcal{A}_{k}$, for each sort $s \in \mathcal{S}_{k}$. Then, models of a MEL theory $\mathcal{E}=(\Sigma, \Gamma)$ are models of $\Sigma$ satisfying the axioms $\Gamma$. There is a sound and complete inference system to derive all theorems of a MEL theory $(\Sigma, \Gamma)$ [2]. We denote the initial algebra of $\mathcal{E}=(\Sigma, \Gamma)$ by $T_{\mathcal{E}}$. There is a unique $\Sigma$-homomorphism $h: T_{\mathcal{E}} \rightarrow A$ for every model $A$ of $\mathcal{E}$.

Under appropriate assumptions on the MEL theory $\mathcal{E}$ the conditional equations can be used from left to right as rewrite rules [3]. This is the way in which MEL is efficiently implemented in the Maude language [8]. An inference system for MEL reasoning is described in detail in Figure 7, page 57 of [3]. The notions of confluence and termination of term rewriting can be generalized to conditional 
MEL theories by corresponding notions of confluence and reductiveness [3]. Since sort computations are involved, a third important notion is sort decreasingness. Assuming that $\mathcal{E}$ is confluent and reductive, we can characterize sort decreasingness as the property that for each term $t$ if we can infer $t: s$ with the rewrite inference system in Figure 7 of [3], then we can also infer $\operatorname{can}_{\mathcal{E}}(t): s$ in the same system. Intuitively, the more we simplify a term with the equations, the easier it becomes to compute its sort without having to remember any intermediate terms in the rewrite computation.

\section{A Partial Specification Example}

In Misra's data type of powerlists [9], a powerlist must be of length $2^{n}$ for some $n \in \mathbb{N}$, and the zip operator $\bowtie$ is only fully defined on powerlists of equal length. We can specify powerlists in MEL as a Maude functional module as follows:

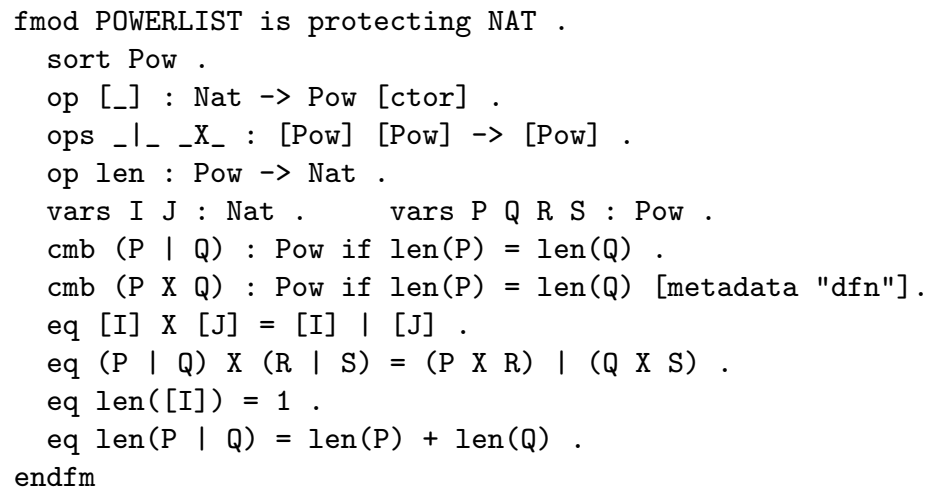

In the functional module POWERLIST, we include the predefined module NAT, which declares the natural numbers with the expected arithmetic operations and relations. In the sort declaration section, we introduce the sort Pow, which we will reserve for those terms representing powerlists; Maude automatically introduces also the kind [Pow] to denote the kind of the sort Pow. In the operator declaration section, we introduce four operators: [_] for representing the operation that forms powerlist elements; _ I _ for representing the powerlist tie operation; _ $\mathrm{X}_{\text {_ }}$ for representing the powerlist zip operation; and len for representing the operation that computes the length of a powerlist. Since we know that not all terms constructed with the operators _ $I_{-}$and _ $\mathrm{X}_{-}$will represent powerlists, we declare those operators at the kind level. For example, [4] $\bowtie([2] \mid[3])$ is not a powerlist. This is represented in POWERLIST by the fact that the term [4] X ( [2] । [3]) has kind $[\mathrm{Pow}]$, but it does not belong to the sort Pow. On the other hand, since we want to use the [_] operator to construct powerlists (in particular, powerlists with only one element), we declare this operator at the sort level and with the ctor attribute. Finally, since we expect that the len operator applied to a powerlist will always evaluate to a natural number, we declare this operator at the sort level, but without the ctor attribute. 
In the variable declaration section, we associate to the variables $I$ and $\mathrm{J}$ the sort Nat, and to the variables $\mathrm{P}, \mathrm{Q}, \mathrm{R}$, and $\mathrm{S}$ the sort Pow. By doing this, we are in fact declaring: $i$ ) that $I$ and $J$ are variables of the kind [Nat], and $P, Q, R$, and $\mathrm{S}$ of the kind [Pow], and ii) that in all memberships and equations in which those variables appear, there is an extra condition stating that those variables only range over the set of terms belonging to their associated sort. Finally, in the membership declaration section, we declare that both the tie and the zip of two powerlists are powerlists if they have equal length; however, since we do not want to use the _ $\mathrm{X}_{-}$operator as a constructor operator for terms representing powerlists, but rather as a defined operator, we declare the membership for the _ $\mathrm{X}_{\text {_ }}$ operator with the dfn attribute. In fact, if we go back to the operator declarations section, we can realize that

op [_] : Nat $\rightarrow$ Pow [ctor]. op len : Pow $\rightarrow$ Nat.

is just syntactic sugar for the following declarations:

op [_] : [Nat] $\rightarrow[\mathrm{Pow}]$. op len : [Pow] $\rightarrow$ [Nat].

mb [I] : Pow. mb len(P): Nat [metadata "dfn"].

As we will explain in the following section, the sufficient completeness problem for POWERLIST reduces to proving that all terms $P$ X $Q$ and len $(P)$, where $P$ and $Q$ are terms built with our constructor memberships, can be proved to be of sort Pow without using the defined memberships.

\section{Sufficient Completeness for MEL Specifications}

The definition of sufficient completeness for MEL specifications is somewhat subtle, in that in its most general form it cannot be given only in terms of a subsignature $\Omega$ of constructors. The point is that, when specifying the conditional memberships for constructor operators in $\Omega$, other nonconstructor function symbols may appear in the condition. This is illustrated in the powerlist example by the conditional membership for the constructor _ $I_{\text {_ }}$ of powerlists. The definition below strictly generalizes that in [3], which ruled out the use of nonconstructor symbols in conditions of constructor memberships.

Definition 1. Let $\mathcal{E}=\left((\mathcal{K}, \Sigma, \mathcal{S}), E \cup M_{<} \cup M_{\Sigma}\right)$ be a $M E L$ specification where $E$ contains the conditional equations, $M_{<}$contains the memberships corresponding to subsort declarations explained below, and $M_{\Sigma}$ contains the conditional memberships specifying the sorts of function symbols in $\Sigma$. Subsort declarations $s<s^{\prime}$ with $s \neq s^{\prime}$ and $s, s^{\prime} \in S_{k}$ for some $k$ are axiomatized by the conditional membership:

$$
(\forall x: k) x: s^{\prime} \text { if } x: s
$$

Finally, we assume that any conditional membership in $M_{\Sigma}$ is of the form:

$$
(\forall \vec{x}) f\left(t_{1}, \ldots, t_{n}\right): s \text { if } t_{1}: s_{1} \wedge \cdots \wedge t_{n}: s_{n} \wedge \mathcal{C}
$$


where $f \in \Sigma, \vec{x}=\operatorname{var}\left(f\left(t_{1}, \ldots, f_{n}\right)\right)$, and $\mathcal{C}$ is a (possibly empty) conjunction of $\Sigma$-equations and $\Sigma$-memberships, $\operatorname{var}(\mathcal{C}) \subseteq \vec{x}$.

Given a subset of memberships $M_{\Omega} \subseteq M_{\Sigma}$, called constructor memberships, we define a constructor subtheory to be the theory $\mathcal{E}_{\Omega}=\left((\mathcal{K}, \Sigma, \mathcal{S}), E \cup M_{<} \cup M_{\Omega}\right)$. Furthermore, we say that $\mathcal{E}$ is sufficiently complete relative to $M_{\Omega}$ iff $\mathcal{E}_{\Omega}$ is such that the unique $\Sigma$-homomorphism

$$
h: T_{\mathcal{E}_{\Omega}} \rightarrow T_{\mathcal{E}}
$$

is an isomorphism. Finally, we define $M_{\Delta}$ to be $M_{\Sigma}-M_{\Omega}$.

Proposition 1. Let $\mathcal{E}=((\mathcal{K}, \Sigma, \mathcal{S}), \Gamma)$ be a MEL specification satisfying the requirements:

(i) $\mathcal{E}$ and $\mathcal{E}_{\Omega}$ are reductive, ground confluent, and ground sort-decreasing.

(ii) Each membership in $M_{\Omega} \cup M_{\Delta}$ is of the restricted form (1).

Then $\mathcal{E}$ is sufficient complete relative to constructor memberships $M_{\Omega}$ iff for each $k \in \mathcal{K}, t, t^{\prime} \in T_{\Sigma, k}$, and sort $s \in \mathcal{S}_{k}$ we have:

$$
\begin{aligned}
\mathcal{E} \vdash t: s & \Longleftrightarrow \mathcal{E}_{\Omega} \vdash t: s \\
\mathcal{E} \vdash t \stackrel{*}{\rightarrow} t^{\prime} & \Longleftrightarrow \mathcal{E}_{\Omega} \vdash t \stackrel{*}{\rightarrow} t^{\prime}
\end{aligned}
$$

where ${ }^{2}$ is MEL inference using the equations as rewrite rules in Figure 7 of [3].

Proof. To see this note that $T_{\mathcal{E}} \cong T_{\mathcal{E}_{\Omega}}$ iff for each $k \in \mathcal{K}, t, t^{\prime} \in T_{\Sigma, k}$, and $s \in \mathcal{S}_{k}$ :

$$
\begin{aligned}
\mathcal{E} \vdash t: s & \Longleftrightarrow \mathcal{E}_{\Omega} \vdash t: s \\
\mathcal{E} \vdash t=t^{\prime} & \Longleftrightarrow \mathcal{E}_{\Omega} \vdash t=t^{\prime}
\end{aligned}
$$

As $\mathcal{E}$ and $\mathcal{E}_{\Omega}$ are reductive, ground confluent, and ground sort-decreasing, we can use the equivance between $\vdash$ and $\vdash$ for such theories and Property 1 and 2 to show:

$$
\begin{gathered}
\mathcal{E} \vdash t: s \Longleftrightarrow \mathcal{E} \vdash t: s \Longleftrightarrow \mathcal{E}_{\Omega} \vdash t: s \Longleftrightarrow \mathcal{E}_{\Omega} \vdash t: s \\
\mathcal{E} \vdash t=t^{\prime} \Longleftrightarrow \mathcal{E} \vdash t=t^{\prime} \Longleftrightarrow \mathcal{E}_{\Omega} \vdash t=t^{\prime} \Longleftrightarrow \mathcal{E}_{\Omega} \vdash t=t^{\prime}
\end{gathered}
$$

Theorem 1. Let $\mathcal{E}=\left(\Sigma, E \cup M_{<} \cup M_{\Omega} \cup M_{\Delta}\right)$ be a MEL specification satisfying the requirements (i) and (ii) in Proposition 1.

Then the two statements below are equivalent:

(a) $\mathcal{E}$ is sufficiently complete relative to constructor memberships $M_{\Omega}$

(b) For each membership $(\forall \vec{x}) t: s$ if $\mathcal{C}$ in $M_{\Delta}$ and ground substitution $\theta: \vec{x} \rightarrow$ $T_{\Sigma}$ such that $\mathcal{E}_{\Omega} \vdash \mathcal{C} \theta$, either $t \theta$ is $\mathcal{E}_{\Omega}$-reducible or there is a membership in $M_{\Omega}$ of the form $(\forall \vec{y}) u: s^{\prime}$ if $\mathcal{C}^{\prime}$ with $s^{\prime} \leq s$ and a substitution $\tau: \vec{y} \rightarrow T_{\Sigma}$ such that $t \theta=u \tau$ and $\mathcal{E}_{\Omega} \Vdash \mathcal{C}^{\prime} \tau$. 
Proof. $(a) \Rightarrow(b)$ : If $\mathcal{E}_{\Omega} \vdash \mathcal{C} \theta$, then we have a fortiori $\mathcal{E} \vdash \mathcal{C} \theta$, and therefore $\mathcal{E} \Vdash t \theta: s$. By Proposition 1 we then have $\mathcal{E}_{\Omega} \Vdash t \theta: s$. As $\mathcal{E}_{\Omega}$ is reductive, ground confluent, and ground sort decreasing, we have $\mathcal{E}_{\Omega} \Vdash t \theta: s$ iff $\mathcal{E}_{\Omega} \Vdash \operatorname{can}_{\mathcal{E}_{\Omega}} t \theta: s$. As a consequence, either $t \theta$ is $\mathcal{E}_{\Omega}$-reducible, or it is already in $\mathcal{E}_{\Omega}$-canonical form, ie. $t \theta=\operatorname{can}_{\mathcal{E}_{\Omega}}(t \theta)$, and in that case a proof of $\mathcal{E}_{\Omega} \vdash t \theta: s$ must necessarily use a membership in $M_{\Omega}$ assigning a sort $s^{\prime}<s$ to $t \theta$.

$(b) \Rightarrow(a)$ : We can use the characterization of sufficient completeness from Proposition 1 . We reason by noetherian induction on $\left(\succ_{\mathcal{E}} \cup \triangleright\right)$. The base case is that of an $\mathcal{E}$-irreducible constant $a$. If $\mathcal{E} \nvdash a: s$, there must be a sort $s^{\prime} \leq s$ and a membership in $M_{\Sigma}$ of the form $a: s^{\prime}$, so that we also have $\mathcal{E}_{\Omega} \vdash a: s^{\prime}$. If the membership $a: s^{\prime}$ is in $M_{\Delta}$, than by hypothesis we must have another membership $a: s^{\prime \prime}$ in $M_{\Omega}$ with $s^{\prime \prime}<s^{\prime}$, so that we get Property 1 . Property 2 also follows trivially by $a$ being in $\mathcal{E}$-normal form.

Consider now a non-constant term $t \in T_{\Sigma}$. We can distinguish two cases: (a) $t$ is in $\mathcal{E}$-normal form, or (b) $t$ is $\mathcal{E}$-reducible. In case (a), to see Property 1, suppose $\mathcal{E} \vdash t: s$. This can only happen if there is a subsort $s^{\prime} \leq s$ and a membership $m=(\forall \vec{x}) u: s^{\prime}$ if $\mathcal{C}$ in $M_{\Sigma}$ such that there is a substitution $\theta: \vec{x} \rightarrow T_{\Sigma}$ with $t=u \theta$ and $\mathcal{E} \models \mathcal{C} \theta$. If $m \in M_{\Omega}$, we are done by noetherian induction and the reductiveness assumption. If not, we also have a proof $\mathcal{E} \Vdash t: s^{\prime}$, and then by the induction hypothesis we have another membership in $M_{\Omega}$ assigning a sort $s^{\prime \prime}<s^{\prime}$ to $t$. Then again by the induction hypothesis we have $\mathcal{E}_{\Omega} \Vdash t: s$. Property 2 follows trivially, since $t$ is in $\mathcal{E}$-normal form. In case $(b)$, the key observation is that, using noetherian induction, all the one-step rewrite proofs $\mathcal{E} \Vdash t \stackrel{1}{\rightarrow} t^{\prime}$ are such that $\mathcal{E}_{\Omega} \Vdash t \stackrel{1}{\rightarrow} t^{\prime}$. Since we then have $t \succ_{\mathcal{E}} t^{\prime}$, the result then follows easily using noetherian induction plus the fact that, by hypothesis, $\mathcal{E}$ is sort-decreasing.

\section{The Maude Sufficient Completeness Tool}

The Maude Sufficient Completeness tool (SCC) is itself written in Maude using reflection. (More details on reflection in Maude in Sect. 5.2.) The soundness of the tool is based on Theorem 1. There are two major components to the tool: a Sufficient Completeness Analyzer, which generates proof obligations for sufficient completeness problems, and the Maude Inductive Theorem Prover (ITP), extended with additional commands to try to automatically prove those proof obligations. The tool has been run on a variety of different MEL specifications, and is available for download with source, documentation, and examples (including MEL specifications of ordered lists with sorting functions, stacks, and binary trees) from the tool's webpage: http://maude.cs.uiuc.edu/tools/scc/

\subsection{The Maude Sufficient Completeness Analyzer}

The Maude Sufficient Completeness Analyzer follows the incremental constructorbased narrowing of patterns approach, but generalized to handle conditional 
specifications. Given a MEL theory $\mathcal{E}=\left(\Sigma, E \cup M_{<} \cup M_{\Sigma}\right)$ in Maude, conveniently annotated to indicate a constructor subtheory $\mathcal{E}_{\Omega}$, the Maude sufficient completeness analyzer generates, in a two phase process, a set of proof obligations which if discharged, ensures the sufficient completeness of $\mathcal{E}$ relative to $M_{\Omega}$. The sufficient completeness analyzer assumes that $\mathcal{E}$ satisfies the requirements (i) and (ii) in Proposition 1.

The Narrowing Procedure. In its first phase, the analyzer returns a set $\Delta=\left\{(t, s, \mathcal{C})_{i}\right\}_{i \in \mathbb{N}}$ such that, if $t^{\prime}$ is a counterexample for sufficient completeness, then there exists a triple $(t, s, \mathcal{C}) \in \Delta$ and a substitution $\theta: \operatorname{var}(t) \rightarrow T_{\Sigma}$ such that $t^{\prime}=\theta t$ and $\mathcal{E}_{\Omega} \models \theta \mathcal{C}$. The set $\Delta$ is generated from the initial set $\left\{(t, s, C) \mid(\forall \vec{x}) t: s\right.$ if $\left.\mathcal{C} \in M_{\Delta}\right\}$ by applying rule (2) below until it cannot be applied any more. The rule (2) uses the expandability relation $\triangleleft$ and the expand function exp which are defined as follows:

Definition 2. Let $t, t^{\prime}$ be terms in $T_{\Sigma}(\vec{x})$ such that $\operatorname{var}(t) \cap \operatorname{var}\left(t^{\prime}\right)=\emptyset$, and $x \in \operatorname{var}(t)$. Then, $t \boldsymbol{\bullet}_{x} t^{\prime}$ iff there exists a substitution $\theta$ such that $\theta$ is a most general unifier of $t$ and $t^{\prime}$, and $\theta(x)$ is not a variable.

Definition 3. Let $t \in T_{\Sigma}(\vec{x})_{k}, s \in \mathcal{S}_{k}, \mathcal{C}$ a conjunction of atomic formulas, $x \in \vec{x}$ with $x: s^{\prime} \in \mathcal{C}$, and $M$ a set of memberships whose variables have all been renamed to be disjoint from $\vec{x}$. Then,

$$
\exp (t, s, \mathcal{C}, x, M)=\left\{\left(t \theta, s, \mathcal{C} \theta \wedge \mathcal{C}^{\prime}\right) \mid(\forall \vec{y}) u: s^{\prime} \text { if } \mathcal{C}^{\prime} \in M, \theta=(x \mapsto u)\right\}
$$

Finally, we define the inference rule that generates the set $\Delta$. Note that this rule will only be applied a finite number of times, because of the condition $t \boldsymbol{\iota}_{x} t^{\prime}$ on the rule.

$\Delta$-rule For any $(\forall \vec{y}) t^{\prime}=t^{\prime \prime}$ if $\mathcal{C}^{\prime}$ in $E$,

$$
\frac{\Delta^{\prime} \cup\{(t, s, \mathcal{C})\}}{\Delta^{\prime} \cup \exp \left(t, s, \mathcal{C}, x, M_{<} \cup M_{\Omega}\right)} \quad \text { if } x \in \operatorname{var}(t), t \boldsymbol{\triangleleft}_{x} t^{\prime}
$$

The Proof Obligation Generator. In its second phase, the SCC produces, from the set $\Delta$, a set of proof obligations that if discharged guarantees that $\mathcal{E}$ is sufficiently complete with respect to $M_{\Omega}$. Since a triple $(t, s, \mathcal{C}) \in \Delta$ represents a set of potential counter examples, the proof obligation generator produces a sentence which if proven in $\mathcal{E}_{\Omega}$ implies that for every substitution $\theta: \operatorname{var}(t) \rightarrow T_{\Sigma}$ at least one of the following holds:

a) $\mathcal{E}_{\Omega} \not \models \mathcal{C} \theta$

b) $t \theta$ is reducible

c) There exists a membership $(\forall \vec{y}) u: s^{\prime}$ if $\mathcal{C}^{\prime}$ in $M_{\Omega}$ with $s^{\prime}<s$ and a substitution $\tau: \vec{y} \rightarrow T_{\Sigma}$ such that $t \theta=u \tau$ and $\mathcal{E}_{\Omega} \models \mathcal{C}^{\prime} \tau$. 
In particular, for each $(t, s, \mathcal{C}) \in \Delta$, the proof obligation generator constructs the sentence:

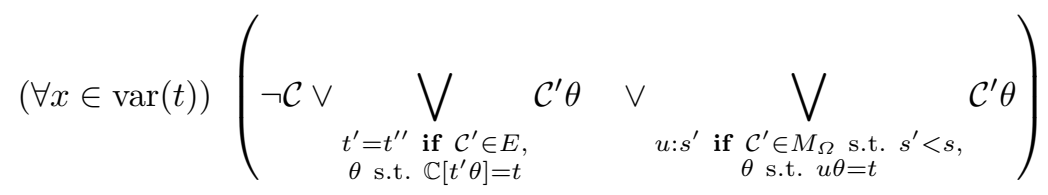

where $\mathbb{C}$ denotes a context.

\subsection{The Maude ITP}

The ITP tool is an experimental interactive tool for proving properties of MEL specifications in Maude. The ITP tool has been written entirely in Maude, and is in fact an executable specification in MEL of the formal inference system that it implements. The ITP inference system treats MEL specifications as data - for example, it may add an induction hypothesis as a new equational axiom. This makes a reflective design, in which Maude equational specifications become data at the metalevel, ideally suited for implementing the ITP. Indeed, the fact that membership equational logic is a reflective logic [10] and that Maude efficiently supports reflective MEL computations is systematically exploited in this tool. A similar reflective design has been successfully adopted to develop other formal tools in Maude [11]. Using reflection to implement the ITP tool has one important additional advantage, namely, the ease to rapidly extend it by integrating other tools implemented in Maude using reflection as it is the case of the SCC.

In the ITP, the user introduces commands that are interpreted as actions that may change the state of the proof. The state of a proof is the set of goals that remains to be proved, each goal consisting of a formula to be proved and the MEL specification in which the formula must be proved. After executing the action requested by the user, the tool reports the resulting state of the proof. The main module implementing the ITP is the ITP-TOOL module. In this module, states of proofs, sets of goals, goals and formulas are represented by terms of different sorts, and the actions interpreting the commands are represented as different, equationally defined functions over those terms.

To integrate the SCC in the ITP we have added a new command scc to the ITP. This command is implemented by extending the module ITP-TOOL with a new, equationally defined function that given an equational specification $\mathcal{E}$ evaluates as follows:

- first, it calls on $\mathcal{E}$ the function checkCompleteness, which implements the sufficient completeness analyzer described in Sect. 5.1;

- then, it converts the resulting proof obligations into a set of ITP goals, which are all associated with $\mathcal{E}_{\Omega}$;

- finally, it eliminates from the state of the proof those goals that can be proved automatically using the ITP auto* command. ${ }^{3}$

${ }^{3}$ The current implementation of the auto* command integrates its rewriting-based simplification strategy with a decision procedure for linear arithmetic with uninter- 
As an example, we can use the scc command to check the sufficient completeness of the POWERLIST functional module.

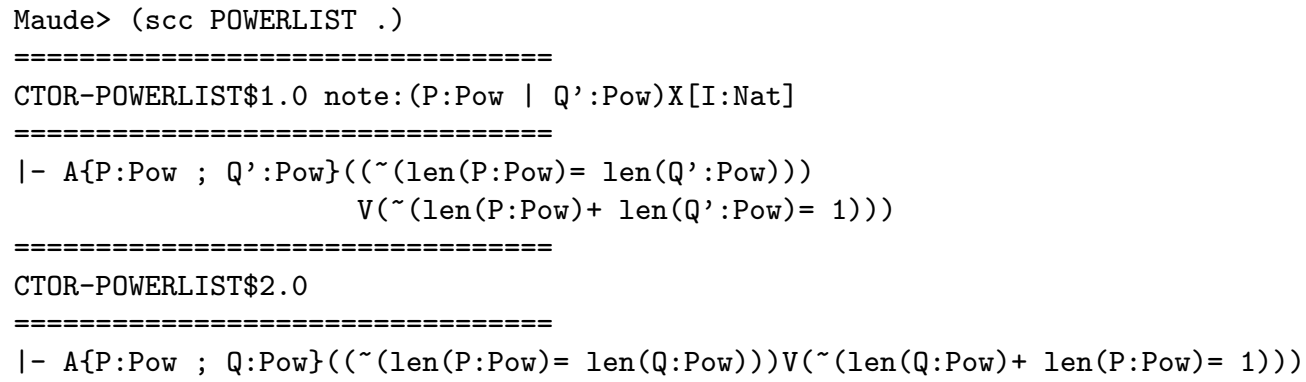

In this case, the auto* command fails to discharge the above goals corresponding to proof obligations generated by SCC, despite the fact that the formulas associated to those goals are obviously true in the $\mathcal{E}_{\Omega}$ The reason is the following. In $\mathcal{E}_{\Omega}$, the len operator is declared at the kind level: it takes a term of the kind [Pow] and returns a term of the kind [Nat]. In this situation, the decision procedure cannot recognize the formulas associated to the above goals as belonging to the class of formulas that it can solve. Therefore, to discharge the proof obligations it is necessary to prove that the operator len always returns a term of sort Nat when it is called on terms of sort Pow. This is, however, implied by the fact that SCC has generated no proof obligations for the len operator. Since this is a rather common situation, we have implemented another command, scc* that associates all the goals corresponding to the proof obligations generated by SCC with $\mathcal{E}_{\Omega}$ but extended this time with all the operator declarations in $\mathcal{E}$ that SCC has found unproblematic. In the case of powerlists, scc* will discharge all the proof obligations automatically.

\section{Related Work}

We cannot survey here the extensive literature on sufficient completeness: we just mention some related work to place things in context. Sufficient completeness of MEL specifications was first studied in [3]; the definition and methods on which the present tool is based are strictly more general than those in [3], allowing a much wider class of MEL specifications to be checked. Sufficient completeness itself goes back to Guttag's thesis [12]; for a good review of the literature up to the late $80 \mathrm{~s}$, as well as some important decidability/undecidability and complexity results, see $[13,14]$. A more recent development is the casting of the decidable cases of sufficient completeness as tree automata decision problems: see Chapter 4 of [15] and references there. Two sufficient completeness tools having a similar approach to ours, namely the incremental constructor-based narrowing of patterns, are the sufficient completeness checkers of the Spike [16]

preted function symbols; this theory includes many of the formulas that one tends to encounter in proof obligations generated by the SCC tools. 
and RRL [17] theorem provers, both of which are based on many-sorted equational logic. By contrast, our approach is based on a more expressive partial equational logic (MEL). However, RRL [17], although based on a total manysorted logic, can address some partiality issues in a different way: incompleteness can be due to omissions, yielding real counterexample patterns, or can be intentional, due to partiality, in which case the partial function's domain of definition can be specified by a quantifier-free formula, which can be used to ascertain if a counterexample pattern is relevant in that domain.

\section{Conclusions and Future Work}

At present, the SCC can handle specifications where some symbols have been declared commutative. Future work will extend the tool to handle equations modulo different combinations of associativity, commutativity, and identity. It is well-known that sufficient completeness is undecidable in the presence of associative axioms, even for left-linear confluent and terminating equations [14]. However, equational tree automata techniques in the style of [18] can still make the problem decidable for some subclasses, and the ITP can support reasoning to discharge proof obligations for the general case.

As already mentioned, the tool assumes MEL specifications $\mathcal{E}$ that are ground confluent, reductive, and sort-decreasing. Although Maude already has tools to check these properties in the special case where $\mathcal{E}$ is an order-sorted specifications [19-21], tools to discharge the corresponding obligations for general MEL specifications need to be developed. For termination of MEL specifications there is already a tool prototype [22] and supporting theory [22,23]. For checking confluence and sort-decreasingness of general MEL specifications detailed supporting theory can be found in [3], but a tool needs to be developed.

\section{References}

1. Martí-Oliet, N., Palomino, M., Verdejo, A.: A tutorial on specifying data structures in Maude (2004) Presented at PROLE'04.

2. Meseguer, J.: Membership algebra as a logical framework for equational specification. In: In 12th International Workshop on Recent Trends in Algebraic Development Techniques (WADT'97). Volume 1376 of Lecture Notes in Computer Science., Springer-Verlag (1998) 18-61

3. Bouhoula, A., Jouannaud, J.P., Meseguer, J.: Specification and proof in membership equational logic. Theoretical Computer Science 236 (2000) 35-132

4. Meseguer, J., Roşu, G.: A total approach to partial algebraic specification. In: Proceedings of ICALP. Volume 2380 of Lecture Notes in Computer Science., Springer (2002) 572-584

5. Meseguer, J., Palomino, M., Martí-Oliet, N.: Equational abstractions. In: Proceedings of CADE. Volume 2741 of Lecture Notes in Computer Science., Springer (2003) 2-16

6. Kapur, D., Subramaniam, M.: New uses of linear arithmetic in automated theorem proving by induction. Journal of Automated Reasoning 16 (1996) 39-78 
7. Clavel, M., Kapur, D., Meseguer, J.: A stronger cover set induction scheme. In preparation (2004)

8. Clavel, M., Durán, F., Eker, S., Lincoln, P., Martí-Oliet, N., Meseguer, J., Quesada, J.: Maude: Specification and programming in rewriting logic. Theoretical Computer Science 285 (2002) 187-243

9. Misra, J.: Powerlist: a structure for parallel recursion. ACM Transactions on Programming Languages and Systems 16 (1994) 1737-1767

10. Clavel, M., Meseguer, J., Palomino, M.: Reflection in membership equational logic, many-sorted equational logic, horn logic with equality, and rewriting logic. In Gadducci, F., Montanari, U., eds.: Proc. 4th. Intl. Workshop on Rewriting Logic and its Applications, ENTCS, Elsevier (2002)

11. Clavel, M., Durán, F., Eker, S., Meseguer, J., Stehr, M.O.: Maude as a formal meta-tool. In Wing, J., Woodcock, J., eds.: FM'99 - Formal Methods. Volume 1709 of Lecture Notes in Computer Science., Springer-Verlag (1999) 1684-1703

12. Guttag, J.: The Specification and Application to Programming of Abstract Data Types. PhD thesis, University of Toronto (1975) Computer Science Department, Report CSRG-59.

13. Kapur, D., Narendran, P., Zhang, H.: On sufficient-completeness and related properties of term rewriting systems. Acta Informatica 24 (1987) 395-415

14. Kapur, D., Narendran, P., Rosenkrantz, D.J., Zhang, H.: Sufficient-completeness, ground-reducibility and their complexity. Acta Informatica 28 (1991) 311-350

15. Comon, H., Dauchet, M., Gilleron, R., Jacquemard, F., Lugiez, D., Tison, S., Tommasi, M.: Tree automata techniques and applications. Available on: http://www.grappa.univ-lille3.fr/tata (1997) release October, 1st 2002.

16. Bouhoula, A., Rusinowitch, M.: SPIKE: A system for automatic inductive proofs. In: Algebraic Methodology and Software Technology, AMAST '95, Proceedings. Volume 936 of Lecture Notes in Computer Science., Springer (1995) 576-577

17. Kapur, D.: An automated tool for analyzing completeness of equational specifications. In: Proceedings of the 1994 International Symposium on Software Testing and Analysis (ISSTA), August 17-19, 1994, Seattle, WA, USA. Software Engineering Notes, Special Issue, ACM Press (1994) 28-43

18. Ohsaki, H., Seki, H., Takai, T.: Recognizing boolean closed a-tree languages with membership conditional rewriting mechanism. In: Rewriting Techniques and Applications, 14th International Conference, RTA 2003, Valencia, Spain, June 9-11, 2003, Proceedings. Volume 2706 of Lecture Notes in Computer Science., Springer (2003) 483-498

19. Clavel, M., Durán, F., Eker, S., Meseguer, J.: Building equational proving tools by reflection in rewriting logic. In: Cafe: An Industrial-Strength Algebraic Formal Method. Elsevier (2000)

20. Durán, F.: Termination checker and Knuth-Bendix completion tools for Maude equational specifications. Technical report, Universidad de Málaga (2000)

21. Durán, F., Meseguer, J.: A Church-Rosser checker tool for Maude equational specifications. Technical report, Universidad de Málaga and SRI International (2000)

22. Durán, F., Lucas, S., Meseguer, J., Marché, C., Urbain, X.: Proving termination of membership equational programs. In: Proceedings of the 2004 ACM SIGPLAN Workshop on Partial Evaluation and Semantics-based Program Manipulation, 2004, Verona, Italy, August 24-25, 2004, ACM Press (2004) 147-158

23. Lucas, S., Meseguer, J., Marché, C.: Operational termination of generalized conditional term rewriting systems. Submitted. (2004) 